\title{
2017 FDA drug approvals: number rebounds but average value slips
}

In line with our outlook last year (Nat Rev. Drug Discov. 16, 78; 2017), there was a rebound in the number of new drugs approved by the FDA in 2017 compared with 2016 (Nat. Rev. Drug Discov. 17, 81-85; 2018). Here, we analyse the 2017 crop of new therapeutic drugs (NTDs), defined as new molecular entities approved by the FDA's Center for Drug Evaluation and Research (CDER) and Center for Biologics Evaluation and Research (CBER), adding combination products with at least one new molecular entity as an active ingredient but subtracting diagnostic imaging agents. As described previously (Nat. Rev. Drug Discov. 13, 331-332; 2014), we use projected peak annual sales of these NTDs as a proxy for value.

In 2017, the FDA approved 52 NTDs with combined projected peak annual sales of US $\$ 47$ billion, substantially higher than the $\$ 32$ billion in 2016 and somewhat above the longer-term mean of $\$ 40$ billion (FIG. 1). Nevertheless, the average forecasted value per approval was lower in 2017 compared with 2016; average value slipped to $\$ 0.9$ billion in 2017 from $\$ 1.1$ billion in 2016, as low as any recent figures excluding 2008-2009. The median value was also down, to $\$ 0.5$ billion in 2017 from $\$ 0.7$ billion in 2016 .
There were three NTDs approved - Sanofi/ Regeneron's dupilumab, Roche's ocrelizumab and Novo Nordisk's semaglutide - with projected peak annual sales of $>\$ 3$ billion, which is on par with 2016. However, in the mid-range, the value of the approved NTDs decreased slightly. Although a single year does not make a trend, we see two reasons for the drop: increased competition in indications and/ or pathways (such as PD1 or PDL1 inhibitors) resulting in lower market share and pressure on prices; and that many of the NTDs address indications with smaller patient numbers (for example, due to genotypic stratification).

NTDs in oncology remained the leading value driver, with $32 \%$ of total projected 2017 peak annual sales, sustaining their long-term (since 2010) average contribution of around one-third. The contribution from drugs outside oncology varies from year to year; in 2017, central nervous system NTDs ranked second.

2017 also saw an increase in approvals of NTDs with breakthrough therapy designation (BTD), with 20 such products (38\% of NTD approvals), up from 7 BTDs (25\%) in 2016. Moreover, these BTDs are expected to generate $65 \%$ ( $>30$ billion) of the total projected peak sales value in 2017, up from $45 \%$ in 2016.
Since 2012, the mean number of NTDs approved annually has been above 40 , compared with 30 in 2000-2011 (FIG. 1). Several factors in addition to the FDA's efforts to grant market authorization quickly on the basis of early efficacy signals support the possibility that this level of output could continue, including improvements in scientific understanding and tools, better internal decision-making and continued focus on underserved specialty and niche indications. There are also system-level risks that could reduce output, however, such as how innovation is rewarded. Nevertheless, in the near-term we believe these risks are diminished and we are cautiously optimistic that 2018 total aggregated peak sales will meet or even exceed the high level in 2017. We also anticipate that the mean value per drug will remain near 2017 levels, given that the pipeline is full of specialty drugs and the growing competition in some indications and pathways.

Mathias Baedeker is at The Boston Consulting Group, Ludwigstraße 21, 80539 München, Germany.

Michael Ringel and Valery Panier are at The Boston Consulting Group, Exchange Place, 31st floor, Boston, Massachusetts 02109, USA.

Ulrik Schulze is at The Boston Consulting Group, Münstergasse 2, 8001 Zürich, Switzerland.

Correspondence to U.S. schulze.ulrik@bcg.com doi: $10.1038 /$ nrd.2018.2 Published online 19 Jan 2018

Competing interests

The authors declare competing interests: see Web version for details.

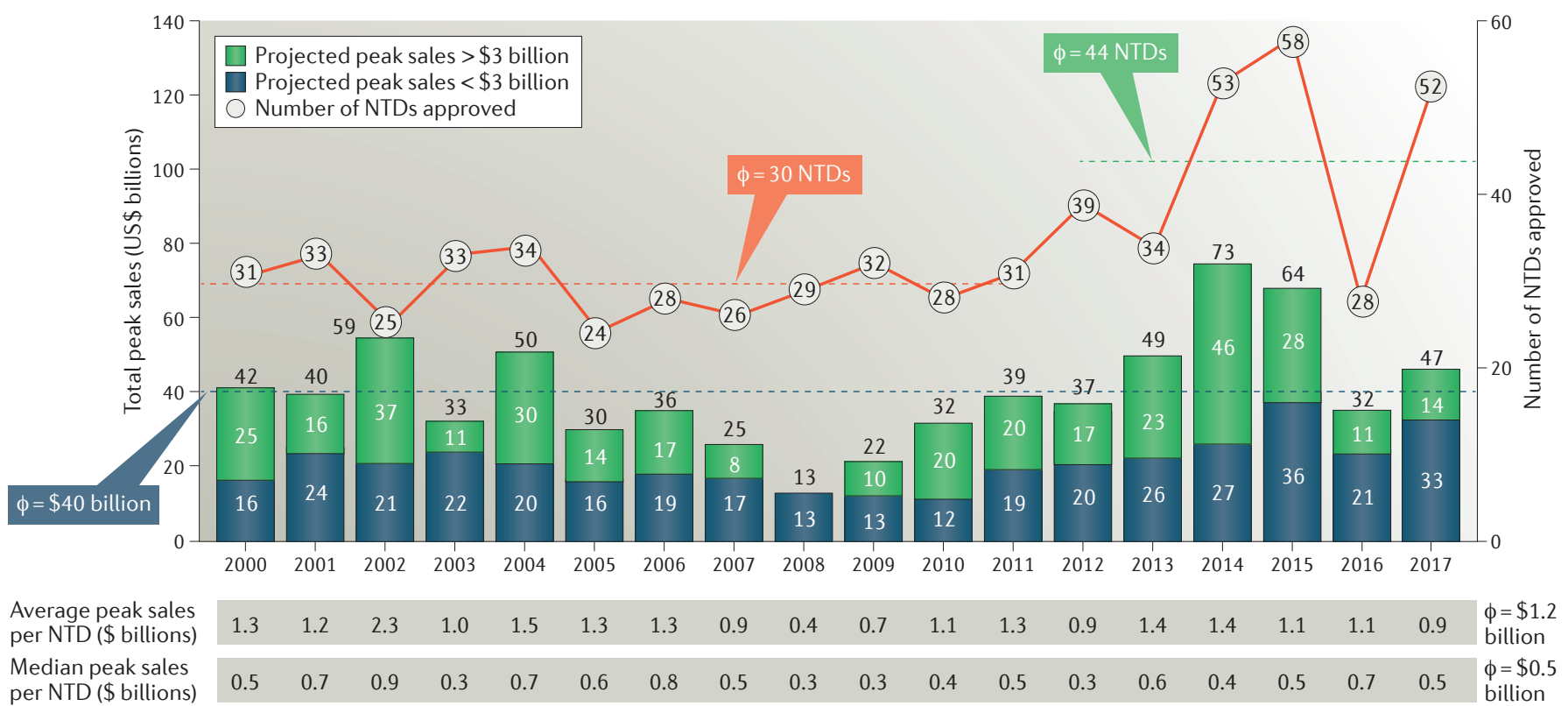

Figure 1 | FDA drug approvals and projected aggregated peak sales: 2000-2017. The graph shows the number and aggregate projected peak worldwide annual sales values of new therapeutic drugs (NTDs) by year of FDA approval. All values are inflation-adjusted to 2017. Because of rounding, not all numbers add up to the totals shown. Sources: EvaluatePharma; FDA; Boston Consulting Group analysis. 